\title{
The importance of bruton tyrosine kinase (BTK) and targeted therapies
}

\begin{abstract}
Abbreviations: BTK, bruton tyrosine kinase; TFKs, tec family kinases; BCR, B-cell antigen receptor; XLA, X-linked agammaglobulinemia; CLL, chronic lymphocytic leukemia; MCL, mantle cell lymphoma; WM, waldenstrom's macroglobulinemia; vWF, von willebrand factor; GPVI, glycol protein VI; CRP, collagen related peptide; $\mathrm{PH}$, pleckstrin homology; TH, TEC homology
\end{abstract}

\section{Introduction}

Bruton Tyrosine Kinase (BTK) is a cytoplasmic non-receptor tyrosine kinase that belongs to the second largest cytoplasmic tyrosine kinase family in the cells of mammalians which is Tec (Tyrosine kinase expressed in Hepato Cellular Carcinoma) Family Kinases (TFKs). ${ }^{1-3}$ The expression of most TFKs including BTK and Tec is predominantly expressed in haematopoietic cells. ${ }^{1,4}$ However Tec has been shown to be detected in other tissues. ${ }^{5}$ BTK plays a central role in signalling through cell surface receptors. It is crucial for B-cell development and differentiation and consider to be a central player in B-Cell antigen Receptor (BCR) signalling. ${ }^{6-8}$ CXCR4 and CXCR5 chemokine receptors, adhesion molecules (integrin), collagen GPVIFCR gamma chain, GPIB-IX-V complex, CLEC-2 signalling and Toll receptor signalling. BTK is primarily expressed in haematopoietic cells, particularly in B cells, but also in monocytes/macrophages, platelets, neutrophils but not $\mathrm{T}$ cells and plasma cells. It is thought that its expression is necessary in the proliferation and survival of B-cells. B-lymphocytes with deficient BTK seem to die at a premature stage. BTK plays a prominent role during B-cell development, as demonstrated in patients with X-Linked Agammaglobulinemia (XLA) who lack peripheral blood B cells and immune globulins, ${ }^{9}$ thus the humoral immune responses cannot be established. ${ }^{1}$ XLA is caused by BTK mutations that result in deficient BTK function. These XLA patients exhibit only a weak platelet aggregation defect in response to low doses of collagen and have no bleeding phenotype. ${ }^{10}$ In the mouse model, the Murine BTK gene mutation ${ }^{8}$ results in X-Linked Immune Deficiency (XID) which is considered to be milder than XLA although the mutation is the same for both. ${ }^{11}$ These differences might be suggestive of species diversity. ${ }^{8,11}$ Several BTK inhibitors have been developed. All BTK inhibitors irreversibly target the C481 residue in the kinase domain of BTK, rendering it kinase-inactive, inducing modest Chronic Lymphocytic Leukemia (CLL) cell apoptosis, abolishing proliferation and BCR signalling (Figure 1). ${ }^{12}$

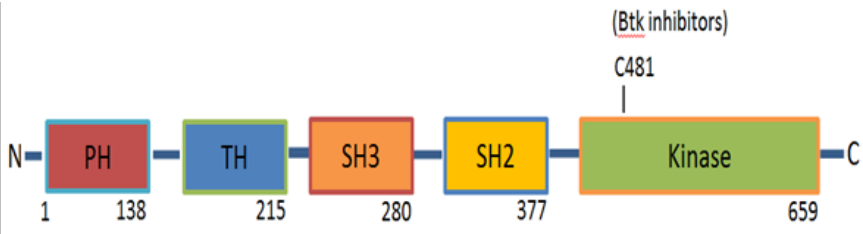

Figure I Structure of BTK. It consists of pleckstrin homology domain (PH), TEC homology $(\mathrm{TH})$ domain, $\mathrm{SH} 3$ domain, $\mathrm{SH} 2$ domain and kinase domain. $\mathrm{C} 48 \mathrm{I}$ which is targeted by BTK inhibitors is in the kinase domain.

\section{BTK targeted therapy}

Ibrutinib (BTK inhibitor) was first synthesised in 2005 and was

\author{
Volume 3 Issue I - 2016
}

\author{
Gasim Dobie,' Denise E Jackson ${ }^{1,2}$ \\ 'Thrombosis and Vascular Diseases Laboratory, RMIT University, \\ Australia \\ ${ }^{2}$ School of Health and Biomedical Sciences, RMIT University, \\ Australia
}

Correspondence: Denise E Jackson, Thrombosis and Vascular Diseases Laboratory, School of Health and Biomedical Sciences, RMIT University, PO Box 7I, Bundoora,Victoria. 3083, Australia, Email denise.jackson@rmit.edu.au

Received: November 17, 2016 | Published: November 18, 2016

tested in humans in 2009. In 2013, Ibrutinib was approved to treat people with B-cell chronic lymphocytic leukemia by food and drug administration in United States (US). ${ }^{13}$ Ibrutinib has significant activity in chronic lymphocytic leukaemia (CLL), Mantle Cell Lymphoma (MCL) and Waldenstrom's Macroglobulinemia (WM). Overall response rates of $>70 \%$ and an estimated 26 month progression free survival rate of $75 \%$. Fast shrinkage of lymph node enlargement lymphocytosis surge and malignant $B$ cell redistribution from sites of the tissue into the peripheral blood are induced by ibrutinib. Majority of patients experience remissions and normal lymphocyte count with continuous therapy with ibrutinib. ${ }^{14}$ The cytoplasmic tyrosine kinase BTK transfers signals from B-cell receptor, tissue homing receptors and other diversity of cell-surface molecules. Its deletion leads to immunodeficiency of B-cells in mice and humans. This in turn, causes the BTK to be the targeted therapy for B-cell disorders (Figure 2).

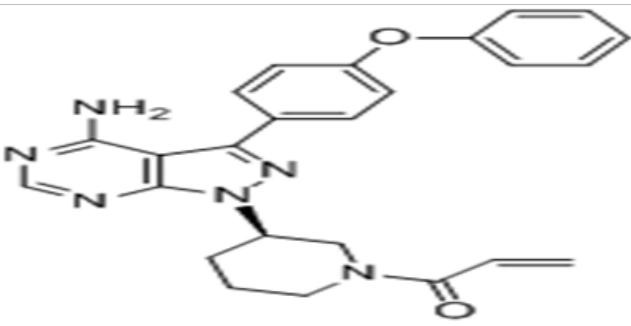

Figure 2 Chemical structure of the BTK inhibitor Ibrutinib (PCl-32765). ${ }^{15}$

Reports of Ibrutinib resistance involving C481S mutation of BTK and gain of function mutations, R665W, L845F and S707Y PLC gamma2 together with adverse side effects have prompted development of next generation of BTK inhibitors. Although Ibrutinib is well tolerated, early clinical studies have reported grade $<2$ bleeding in severity and infrequently with grade $>3$ bleeding events. ${ }^{16}$ Study of function of platelets and clinical histories is obtained to investigate the relationship between ibrutinib and clinical bleeding and its effect on platelet function. On in vitro and ex vivo studies, ibrutinib inhibited aggregation of the platelets either by collagen or GPVI selective ligand, collagen-related peptide. At high shear stress, ibrutinib inhibited adhesion of the platelets to a von Willebrand factor-coated matrix. All patients with sudden epistaxis or bruising experienced 
strong inhibition in platelet aggregation and adhesion in response to collagen suggesting that ibrutinib inhibits platelet function and increases the risk for bleeding. Due to persistence of impaired platelet function over the platelet life span, platelet transfusions might be recommended to re-establish normal haemostasis. ${ }^{17}$ On ex vivo experiment, previous study showed that ibrutinib affects collagen and ADP-dependent platelet activation when added to patient samples. The vital role of BTK in glycoprotein VI signalling is thought to be the reason behind the collagen defect whereas the mechanistic defect in ADP is less clear. ${ }^{18} \mathrm{Kamel}$ et al. ${ }^{18}$ performed light transmission aggregometry determine the effects of BTK on platelets. All patients receiving ibrutinib showed inhibition in collagen-mediated platelet aggregation when tested one week after cessation of the ibrutinib and relapsed one week after restarting the treatment which was a reversible defect on drug cessation. However, on in vivo experiment, no ADP defects were found when using standard doses of the drug. Determining the severity of platelet impairment can also be established with these results using light transmission aggregometry as a tool. ${ }^{18}$ Bruton tyrosine kinase (ibrutinib) also demonstrated effects on collagen, fibrinogen as well as von Willebrand factor. Collagen receptor glycoprotein VI signalling pathway is inhibited with ibrutinib. ${ }^{17}$ It is found that ibrutinib caused milder deficiency in collagen signalling when adhered to immobilised collagen compared to signalling in suspension. It is also found that unstable thrombi were formed when ibrutinib-treated platelets adhered to collagen under arterial flow. ${ }^{19}$ This result suggest that the predominant reason of bleeding in vivo might not be related to deficiency of collagen signalling caused by ibrutinib. However, ibrutinib inhibited platelet signalling when adhered to immobilised fibrinogen suggesting an effect of integrin outside-in $\alpha \operatorname{IIb} \beta 3$ signalling in addition to GPVI signalling. ${ }^{19}$ Combining ibrutinib with $\mathrm{P}_{2} \mathrm{Y}_{12}$ inhibitor inhibited thrombus formation additively under arterial flow. ${ }^{19}$ Ibrutinib also affected vWF-mediated platelet activation under arterial flow. ${ }^{17}$ The tendency of bleeding correlates with decreased collagen-induced platelet aggregation and firm adhesion of platelets on von Willebrand Factor (vWF) under arterial flow. ${ }^{17}$ In vitro results show that ibrutinib inhibited human platelet activation and aggregation, BTK phosphorylation, spreading on fibrinogen and exposure of P-selection under conditions of shear flow. However, no prolonged bleeding was seen in bleeding model. ${ }^{20}$ Grade of $\leq 2$ and occasionally grade $\geq 3$ bleeding related adverse events are associated with ibrutinib used for chronic lymphocytic leukemia patients. Platelet function tests in combination with coagulation factors were assessed to investigate and determine the mechanisms behind bleeding and to establish patients who are at risk. 24 months was the median follow up and 49 days was the median time to event. $55 \%$ out of 85 patients recorded grade $\leq 2$ and no grade of $\geq 3$ bleeding events was recorded. Stability of increasing event incidence by 6 months and normalising of von Willebrand factor and factor VIII levels while they were high at baseline suggested that continued therapy decreases the risk of bleeding. All CLL patients (ibrutinib-treated and non ibrutinib treated) showed a reduction in platelet aggregation in response to collagen and ADP compared to normal control with mild further decrease on ibrutinib in response to collagen and improved response to ADP. These results in addition to lower level of factor VIII and von Willebrand factor and prolonged epinephrine closure time conclude that the risk of bleeding is influenced at both treatment-related factor reduction as well as the disease. ${ }^{16}$ Mantle-cell lymphoma and chronic lymphocytic leukemia can be administered effectively with ibrutinib however; bleeding is a subsequent complication of the drug use. Given that most patients with CLL are elderly, many who are candidates for Ibrutinib therapy will have comorbidities requiring anticoagulation and/or anti platelet therapy. The combined use of Ibrutinib with aspirin, NSAIDs and/or clopidogrel could lead to adverse events including haematomas, ecchymoses and gastrointestinal bleeding. Understanding the mechanism of how Ibrutinib may contribute to bleeding compared to other BTK inhibitors and identifying possible predictors for such adverse events is important for clinical management. It is established that BTK plays a role in glycoprotein signalling in platelets through GPIB, GPVI and CLEC2. However the clinical significance of BTK inhibition in platelets is less clear. Recent studies have shown that BTK plays a crucial role during activation of platelets by CRP and collagen by regulating tyrosine phosphorylation and activation of PLC-gamma2. BTK is not essential for thrombin-mediated or ADP-mediated platelet activation. Platelets have a large number and range of receptors which mediate activation during haemostasis. This may explain why XLA patients don't bleed despite the partial loss of their response to collagen. Tec may compensate for the lack of BTK in this clinical scenario. In contrast, GPVI-deficient patients normally have mild bleeding defects and occasionally suffer from severe, acute bleeding events. Besides $\mathrm{BTK}$, Ibrutinib can also inhibit Tec (in vitro $\mathrm{IC}_{50}=78 \mathrm{~nm}$ ), BLK and ITK although its major target is BTK. The combined action of Ibrutinib on GPVI, CLEC-2 and GPIB pathways likely explains the defect in primary haemostasis. These observations raise questions about the biochemical mechanism by which BTK regulates PLC gamma2 tyrosine phosphorylation and activation, and the mechanism by which BTK tyrosine phosphorylation and activation is regulated. This will be important in our understanding of ITAM-mediated signalling in platelets, in particular the mechanisms of regulation of PLC gamma2, BTK and Tec Family Kinases in platelets and other haematopoietic cells. X-linked Agammaglobulinemia (XLA) which is an inherited disease caused by BTK gene mutation and characterised by $\mathrm{B}$ cell maturation arrest at pre-B cell stage. According to experiments done by Alex and his group, embryonic stem cells and BTK mutated gene mice were used to analyse the role of BTK in B cell development. The results indicated that BTK has no role in signalling of pre-B cell receptor in mice whereas signalling of BTKmediated B cell seems to be essential for immature B cells to survive in bone marrow. ${ }^{21}$ It is reported that BTK is crucial for signalling in platelets by collagen receptor Glyco Protein VI (GPVI). Collagen and Collagen-Related Peptide (CRP) are shown to induce tyrosine phosphorylation of BTK in platelets. XLA patients' platelets showed a significant reduction in aggregation, metabolism of calcium and secretion of dense granules in response to collagen and CRP while no significant alteration in aggregation and secretion in response to thrombin. These results illustrate the importance of BTK in collagen activation via GPVI/FCR gamma-chain (ITAM) signalling pathway but do not have a role in thrombin-mediated (G protein coupled) activation of platelets. $^{9}$

\section{Acknowledgements}

None.

\section{Conflict of interest}

The author declares no conflict of interest.

\section{References}

1. Mohamed AJ, Yu L, Bäckesjö CM, et al. Bruton's tyrosine kinase (Btk): function, regulation and transformation with special emphasis on the $\mathrm{PH}$ domain. Immunol Rev. 2009;228(1):58-73. 
2. Smith CI, Islam TC, Mattsson PT, et al. The Tec family of cytoplasmic tyrosine kinases: mammalian Btk, Bmx, Itk, Tec, Txk and homologs in other species. Bioessays. 2001;23(5):436-446.

3. Rodriguez JG, Readinger JA, Viorritto IC, et al. Tec kinases, actin and cell adhesion. Immunol Rev. 2007;218:45-64.

4. Berg LJ, Finkelstein LD, Lucas JA, et al. TEC family kinases in T lymphocyte development and function. Annu. Annu Rev Immunol. 2005;23:549-600.

5. Mano H, Ishikawa $\mathrm{F}$, Nishida J, et al. A novel protein-tyrosine kinase, tec, is preferentially expressed in liver. Oncogene. 1990;5(12):1781-1786.

6. Vetrie D, Vorechovský I, Sideras P, et al. The gene involved in X-linked agammaglobulinaemia is a member of the src family of protein-tyrosine kinases. Nature. 1993;361(6409):226-233.

7. Tsukada S, Saffran DC, Rawlings DJ, et al. Deficient expression of a B cell cytoplasmic tyrosine kinase in human X-linked agammaglobulinemia. Cells. 1993;72(2):279-290.

8. Thomas JD, Sideras P, Smith CI, et al. Colocalization of X-linked agammaglobulinemia and X-linked immunodeficiency genes. Science. 1993;261(5119):355-358.

9. Quek LS, Bolen J, Watson SP. A role for Bruton's tyrosine kinase (Btk) in platelet activation by collagen. Curr Biol. 1998;8(20):1137-1140.

10. Oda A, Ikeda Y, Ochs HD, et al. Rapid tyrosine phosphorylation and activation of Bruton's tyrosine/Tec kinases in platelets induced by collagen binding or CD32 cross-linking. Blood. 2000;95(5):1663-1670.

11. Vihinen M, Iwata $\mathrm{T}$, Kinnon $\mathrm{C}$, et al. BTKbase, mutation database for X-linked agammaglobulinemia (XLA). Nucleic Acids Res. 1998;24(1):160-165.
12. Woyach JA, Furman RR, Liu TM, et al. Resistance mechanisms for the Bruton's tyrosine kinase inhibitor ibrutinib. $N$ Engl J Med. 2014;370(24):2286-2294.

13. Smith MR. Ibrutinib: a force with a dark side? Blood. 2016;127(12):15231524.

14. Burger JA, Buggy JJ. Bruton tyrosine kinase inhibitor ibrutinib (PCI32765). Leuk Lymphoma. 2013;54(11):2385-9231.

15. Pan Z, Scheerens H, Li SJ, et al. Discovery of selective irreversible inhibitors for Bruton's tyrosine kinase. Chem Med Chem. 2007;2(1):5861

16. Lipsky $\mathrm{AH}$, Farooqui $\mathrm{MZ}$, Tian $\mathrm{X}$, et al. Incidence and risk factors of bleeding-related adverse events in patients with chronic lymphocytic leukemia treated with ibrutinib. Haematologica. 2015;100(12):15711578 .

17. Levade M, David E, Garcia C, et al. Ibrutinib treatment affects collagen and von Willebrand factor-dependent platelet functions. Blood. 2014;124(26):3991-3995.

18. Kamel S, Horton L, Ysebaert L, et al. Ibrutinib inhibits collagen-mediated but not ADP-mediated platelet aggregation. Leukemia. 2015;29(4):783787.

19. Bye AP, Unsworth AJ, Vaiyapuri S, et al. Ibrutinib Inhibits Platelet Integrin $\alpha \operatorname{IIb} \beta 3$ Outside-In Signaling and Thrombus Stability But Not Adhesion to Collagen. Arterioscler Thromb Vasc Biol. 2015;35(11):2326-2335.

20. Rigg RA, Aslan JE, Healy LD, et al. Oral administration of Bruton's tyrosine kinase inhibitors impairs GPVI-mediated platelet function. Am J Physiol Cell Physiol. 2016;310(5):C373-C380.

21. Maas A, Hendriks RW. Role of Bruton's tyrosine kinase in B cell development. Dev Immunol. 2001;8(3-4):171-181. 